\title{
REPERTORIO BIBLIOGRÁFICO CASTELLANO DE MAX WEBER
}

\author{
Julio Cabrera Varela \\ (Universidad de Santiago de Compostela)
}

De un tiempo a esta parte la figura de Max Weber está resurgiendo, aunque tímida y aisladamente por el momento, del lamentable olvido que en nuestro país la envolvía. Siempre es difícil determinar las causas de las frecuentes idas y venidas, olvidos y recupetaciones a que están expuestos los clásicos de la historia del pensamiento occidental; finalmente casi siempre se recurre a la moda intelectual, importada o autóctona, que, como los "Doctores de la Santa Madre Iglesia», puede dar razón de tal o cual resu. rrección. No es éste el caso de Weber, no hay un «Boom Max Weber»; se trata tmás bien del lento reencuentro con un hombre del que, equivocadamente, alguien, con buena o mala fe, había voceado su defunción. La lenta pero profunda crisis que, por inanición, está padeciendo el positivismo sociológico y la necesidad de una nueva refiexión politica, liberada ya de la urgencia del combate diario, propician el que algunas, pocas aún, cabezas pensantes de este país dirijan su mirada sobre la extensa y rica obra de Weber.

Así, en el amplio espectro temático que uno puede imaginar, desde un discurso de apertura parlamentaria hasta los específicos problemas de la organización industrial, es normal encontrarse con numerosas, aunque breves, referencias a su obra. Desgraciadamente este feliz reencuentro con Weber no está acompañado - salvando las honrosas excepciones de sus buenos, pero escasos, conocedores- del tigor y la precisión que él se metece. No hay que impacientarse, todo llegará. En esta paciente espera, el presente repertorio bibliogtáfico quiere ser un instrumento de ayuda para lectores no iniciados en la obra weberiana. 
Como es sabido, la primera traducción íntegra del alemán de la voluminosa Economía y Sociedad fue realizada en 1944 al castellano, mientras que otros idiomas tuvieron que esperar algo más (la primera traducción completa al inglés data de 1968, al italiano de 1961 y al francés de 1971). Las grandes perspectivas que esta edición auguraba para los estudiosos castellanoparlantes se quedaron en «agua de borrajas». Las siguientes traducciones tuvieron un carácter desordenado, parcial, fragmentario, descontextualizado y, casi siempre, siguieton esquemas editoriales importados.

La primera consecuencia de este desorden es la falta de precisión y acuerdo a la hora de citar dichas obras en castellano. Así es normal encontrarse con que una cita tiene por toda referencia la página correspondiente a determinada antología, como si ésta gozase de una unidad temática y cronológica, como es el caso de las ediciones alemanas, cuando en realidad no es sino un refrito de diversos escritos de carácter heterogéneo. De esta forma se pierde la dimensión histórica de los escritos weberianos, tan importante para su comprensión. Por otra parte, esta dinámica de fragmentación provoca el desconocimiento y el desconcierto ante lo que realmente está traducido, y pasan desapercibidos, las más de las veces, textos de gran relevancia que, ya por su cambio de título ya por su forzado matidaje con otros textos, terminan perdiendo su identidad.

Por todo ello me ha parecido necesario establecer claramente la procedencia exacta de cada texto, de manera que facilite el manejo de la bibliografía existente en castellano, conociendo su correspondencia exacta con el original alemán. De esta forma intento agilizar cualquier consulta que desde la referencia en alemán busque la correspondencia castellana y viceversa, desde la edición castellana intente localizar el texto exacto alemán.

Para ello las obras se ordenan cronológicamente según su fecha de publicación original en alemán, independientemente de su agrupación en los diversos Gesammelte, señalando los datos completos de dicha publicación, o, si ésta fue posterior a la muerte de Weber, de su redacción. A continuación figura la paginación correspondiente a la publicación en los Gesammelte y, ya al final, los datos correspondientes a su edición o ediciones en castellano.

En segundo lugar establezco un cuadro de correspondencias entre la paginación de los Gesammelte y sus traducciones correspondientes, facilitando de esta forma la localización castellana, si existiese, de las citas realizadas según dicha ordenación admitida internacionalmente.

$Y$, ya para finalizar, se incluye una bibliografía de cien títulos en castellano, entre libros y artículos, en los que bajo diversos aspectos se estudia la figura de Weber. En este caso, y para facilitar su clasificación 
temática, he preferido desglosat en artículos independientes las diversas compilaciones manejadas. La lista, si bien no es exhaustiva, recoge lo más televante y asequible de las publicaciones en castellano. En este sentido he preferido tefejar el mayor número posible de enfoques (desde un punto de vista sociológico), aunque algunas obras de las citadas traten a Weber secundariamente, y no cumplir con el prejuicio académico de agotar la totalidad de la bibliografía existente.

\section{Abreviatures usades}

GAR, I, II, III: Gesammelte Aufsätze zur Religionssoziologie, Ed. J.C.B. Mohr (Paul Siebeck) Tübingen, 1978 (séptima edición). Primera edición, tomo I, 1920; tomo II, 1921; tomo III, 1920.

GPS: Gesammelte Politische Schriften, Ed. J.C.B. Mohr (Paul Siebeck) Tübingen, 1980 (cuarta edición). Primera edición, 1921.

WL: Gesammelte Aufsätze zur Wissenscbaftslebre, Ed. J.C.B. Mohr (Paul Siebeck) Tübingen, 1973 (cuarta edición). Primera edición, 1922.

WG: Wirtscbaft und Gesellschaft, I, I, III: Ed. J.C.B. Mohr (Paul Siebeck) Tübingen, 1976 (quinta edición). Primera edición, 1922.

ASS: Archiv für Sozialwissenschaft und Sozialpolitik, revista coeditada por Weber, Jaffé y Sombart, desde 1903 y en la que Weber publicará numerosos artículos.

EMS: Ensayos sobre metodologia sociológica, Ed. Amorrortu, Buenos Aires, 1978 (segunda edición). Traducción del original alemán de José Luis Etcheverry.

ES: Economia y Sociedad, Ed. Fondo de Cultura Económica, México, 1979 (sexta edición en un solo volumen). Traducción de la cuatta edición alemana de J. Medina Echavarría, J. Roura Parelle, E. Imaz, E. García Máynez y J. Ferrater Mora.

ESC: Ensayos de Sociologia Contemporánea, Ed. Martínez Roca, S. A., Barcelona, 1972. Es la edición castellana de la selección de textos realizada por H. H. Gerth y C. Wright Mills y publicada con el título: From Max Weber: Essays in Sociology, Oxford University Press, Nueva York, 1946. Traducción al casteliano del original inglés de Mireia Bofill.

ESR. 1: Ensayos sobre sociología de la religión. X.; Ed. Taurus, Madrid, 1983. Traducción de José Almaraz y Julio Carabaña.

EP: Escritos Politicos, I, II, Ed. Folios Ediciones, S. A., México, 1982. Edición a cargo de J. Aricó. Traducción del alemán, italiano e inglés de $F$. Rubio Llorente, E. Molina y Vedia, Romero Medina y Adriana Sandoval. 
«Papers»: Revista de Sociologia

\section{CoRrespondencia biBLIOGRÁfica dE lAs OBRAS DE WEBER TRADUCIDAS AL CASTELLANO}

1891: Die römiscbe Agrargeschichte in ibrer Bedeutung fïr das Staats und Privatrecht, Stuttgart, F. Enke, 1891.

Edición castellana: Historia Agrario Romana, Ed. Akal, Madrid, 1982. Traducción del alemán de V. A. Goazález.

1895: «Der Nationalstaat und die Volkswirtschaftspolitik» (lección inaugural), Friburgo, 1895.

Incluido en GPS, pp. 1.25.

Edición castellana: «El Estado Nacional y la política económica», en EP, tomo I, pp. 3-29.

1896: «Die Sozialen Gründe des Untergangs der ontiken Kultur», en Die Wabrbeit, vol. 6, Stuttgart, 1896, pp. 57-77.

Edición castellana: «La decadencia de la cultura antigua» en «Revista de Occidente», XIII (1926), pp. 25.59.

1904: «Die "Objektivität" sozialwissenschattlicher und sozialpolitischer Er. kenntmis», en ASS, vol. 19 (1904), pp. 22-87.

Incluido en WL, pp. 146-214.

Edición castellana: "La "objetividad" cognoscitiva de la ciencia social y de la polftica social», en EMS, pp. 39-101. Hay otra edición en Max Weber, Sobre la teorió de los ciencias sociales, Ediciones Península, Barcelona, 1974 (segunda edición), traducción de Michael Faber-Kaiser.

*1904-1905: Die protestantische Etbik und der «Geist» des Kapitalismus, en ASS, vols. $20-21$, pp. 1.54 y $1-110,1904$ y 1905.

Incluido en GAR, tonzo I, pp. 17-206.

Edición castellana: La ética protestante y el espiritu del capitalismo, Edicio. nes Península, Barcelona, 1969; traducción del alemán de Luis Legaz Lacamba. Incluye como introducción la «Vorbemerkung» redactada por Weber para el conjunto de GAR, pp. 1-16.

También: ESR, 1: pp. 17-206.

1906: «Kritische Studien auf dem Gebiet der Kulturwissenschaftichen Logik», en ASS, vol. 22, 1906, pp. 143-207.

Incluido en WL, pp. 215.290.

Edición castellana: «Estudios ctíticos sobre la lógica de las ciencias de la cultura», en EMS, pp. 102-174.

1906: «Zur Lage der bürgerlicben Demokratie in Russland», en ASS, vol. 22 (suplemento), 1906, pp. 234-253.

Incluido en GPS, pp. 33-68.

Edición castetlana: "La situación de la democracia burguesa en Rusia», en

EP, tomo II, pp. 365-402.

1906: «Russlands Ubergang zum Scbeinkonstitutionalismus», en ASS, vol. 23, 1906, pp. 165-401.

Incluido en GPS, pp. 69.111. 
Edición castellana: «La transición al seudoconstitucionalismo en Rusia», en EP, tomo II, pp. 403-444.

*1906: «Die protestantischen Sekten und der Geist des Kapitalismus», publicado por primera vez con el título "Kircben" und "Sekten" en Frankfurter Zeitung de 13 y 15 de abril de 1906; luego retomado y ampliado, fue publicado con el título "Kirchen" und "Sekten" in Nordamerika", en Cbristliche Welt, año 20, núm. 24 (14-IV-1906), pp. 558-562 y año 20, núm. 25 (21-IV-1906), pp. 577-583.

Incluido con el título que fagura en primer lugar en GAR, tomo I, pp. 207 236.

Edición castellana: «Las sectas protestantes y el espíritu del capitalismo», en ESC, pp. $370-394$.

También: ESR. 1: pp. 169-192.

1906: «Deutscbe Agrarprobleme in Vergangenbeit und Gegenwart», este título corresponde a una conferencia pronunciada por Weber en 1904 en la Exposición Universal de San Luis, el texto original parece estat perdido; sin embargo se conserva una traducción al inglés con el título *The Relations of the Rural Community to otber Brancbes of Social Sciences, realizada por C. W. Seidenadel para Congress of Arts and Science, Universal Exposition, St. Louis (Boston y Nueva York, Houghton-Mifflin, 1906), vol. VII, páginas 752.746 .

Edición castellana: «Capitalismo y sociedad rural en Alemania», en ESC, pp. 443-470.

1908: «Georg Simmel als Soziolog und Theoretiker der Geldwirtschatt», recensión inconclusa e inédita de la obra de Simmel: Soziologie. Permaneció desconocida hasta 1972, en que fue descubierta por el Instituto Max Weber de Munich y reproducida por primera vez en inglés como «Georg Simmel as sociologist, by Marx Webers en Social Researcb, vol. 39, núm. 1 (1972), pp. 155-163. La fecha del original está sin determinar defnitivamente, pero se supone que data de 1908, fecha de publicación de la obra de Simmel. Edición castellana: «La sociologia de Simmel», en «Papers», núm, 15 (1981), pp. 147-151; editado por Ediciones Península, Barcelona.

1913: «Uber einige Kategorien der verstebenden Soziologie», en Logos. Internationale Zeitschrift für Pbilosopbie der Kultur, vol. IV, 1913, pp. 253-294. Incluido en WL, pp. 427-474.

Edición castellana: «Sobre algunas categorias de la sociología comprensiva», en EMS, pp. 175.221.

1916: «Zwischen zwei Gesetzen", en Die Frau, 1916.

Incluido en GPS, pp. 142.145.

Edición castellana: «Entre dos leyes», en EP, tomo I, pp. 30-34.

*1916: «Die Wirtschattsethik der Weltreligionen. Einleitung, I. Kontuzianismus und Taoismus; Zwiscbenbetrachiung: Theorie der Stufen und Richtungen religiöser Weltablebrung", en ASS, vol. 41, 1916, pp. 1-87 y 335-421. Incluido en GAR, tomo I, pp. 237-573.

Edición castellana: ESR, 1: pp. 136-466. También en castellano se encuen- 
tran traducidas algunas partes distribuidas de la siguiente manera: pp. $237-$ 268 (GAR), traducidas como «Psicología social de las grandes religiones» en ESC, pp. 327-369; pp. 269-394 sin traducir; pp. $395-430$ por «los literatos chinos» en ESC, pp. 507-541; pp. $431-535$ sin traducir; pp. $536-573$ por «Negaciones religiosas del mundo y sus orientaciones», en ESC, pp. 395-439. Hay que señalar que en estas tradicciones los pátrafos, a veces, aparecen dispuestos de manera distinta al original.

1916: «Deutschland unter den europäiscben Weltmächten», resumen de una conferencia pronunciada en Munich el 27-X-1916 y publicada en Die Hilfe, núm. 22, 1916, pp. 735-741.

Incluido en GPS, pp. 157-177.

Edición castellana: "Alemania entre las grandes potencias europeas», en EP, tomo I, pp. 35-58.

1916-1917: «Die Wirtschaftsetbik der Weltreligionen. II. Hinduismus und Buddbismus», en ASS, vol. 41, 1916, pp. 613-744; vol. 42, 1916/1917, pp. 345-461 y 678-814.

Incluido en GAR, tomo I1, pp. 1-378.

Edición castellana: se trata también ésta de una traducción parcial, como sigue: pp. 1.31 (GAR) sin traducir; pp. 32.48 como «India: el bracman y las castas» en ESC, pp. 483-502; pp. $48-51$ sin traducir; pp. 51.57 traducidas en R. Bendix y S. M. Lipset, Clase, Status y Poder, Ed. Euramérica, Madrid, 1972, tomo I, pp. 121-127 como «Casta y "sib" $\gg ;$ pp. 57-108 sin traducir; pp. $109-113$ traducidas en el artículo señalado de ESC en las páginas 502-506; el resto permanece sin traducir.

1917: «Russlands Utbergang zur Scbeindemokratie», en Die Hilfe, núm. 23, de 26-IV-1917, pp. 272-279.

Incluido en GPS, pp. 197-215.

Edición castellana: «La transición de Rusia a la seudodemocracia», en EP, tomo H, pp. $445-469$.

1917: "Der Sinn der "Wertreibeit" der Soziologiscben und okonomischen Wissenscbaften", en Logos, vol. VII, 1917, pp. 40-88.

Incluido en WL, pp. 489-540.

Edición castellana: «El sentido de la "neutralidad valorativa" de las cien. cias sociológicas y económicas», en EMS, pp. 222.269. Ha sido publicado también en Max Weber, Sobre la teoria de las ciencias sociales, Ediciones Península, Barcelona, 1974 (segunda edición), traducción de Michael FaberKaiser.

1917: «Vaterland und Vaterlandspartei», en Mincbener Neueste Nacbricbten del 30-IX-1917.

Incluido en GPS, pp. 229-232.

Edición castellana: «La patria y el partido de la pattia», en EP, tomo I, pp. 163-166.

1917: «Wablrecht und Demokratie in Deutscbland», en Der Deutsche Volksstaat. Scbriften zur inneren Politik. Editado por Wilh. Heile y Walther Schote, núm. 2, diciembre de 1917. 
Inciuido en GPS, pp. 245-291.

Edición castellana: «Sistema electoral y democracia en Alemania», en EP, tomo 1, pp. 167-217.

Hay también una traducción parcial en ESC, pp. 471-482 correspondiente a las pp. 277-287 de GPS con el título «El carácter nacional y los Junkers».

1918: «Parlament und Regierung im neugeordneten Deutscbland», serie de artículos publicados en 1917 en Frankfurter Zeitung, recopilados y publicados por Duncker u. Humboldt, Munich, 1918.

Incluidos en GPS, pp. 306.443.

Edicion castellana: "Parlamento y gobierno en el nuevo ordenamiento alemán», en EP, tomo I, pp. 59-162. (Excluye el último apartado que com* prende las páginas $406-443$ de GPS.)

1918: «Der Sozialismus», conferencia pronunciada en Viena en julio de 1918; publicado en Viena, 1918.

Edición castellana: «El socialismo», en EP, tomo II, pp. 219-252.

1918: «Deutscblands kïnftige Staatsform», serie de artículos publicados en 1918 en Frankfurter Zeitung y recopilados y reeditados en volumen separâdo en la misma revista en el núm. 2 de 1919.

Incluido en GPS, pp. 448-483.

Edición castellana: «La futura forma institucional de Alemania», en EP, tomo II, pp. 253-293.

1918: «Das neue Deutscbland», crónica de un discurso pronunciado por Weber el 1 de diciembre de 1918 en Frankfurt en favor del Partido Demócrata Alemán, y publicado en una edición especial del Frankfurter Zeitung de la misma fecha.

Inciuido en GPS, pp. 484-487.

Edición castellana: «La nueva Alemania», en EP, tomo II, pp. 294-297.

1919: "Der Reicbspräsident», en Berliner Börsenteitung, 25 de febreto de 1919.

Incluido en GPS, pp. 498-501.

Edición castellana: «El presidente del Reich», en EP, tomo IX, pp. 303-307.

1919: «Die Untersucbung der Scbuldtrige», en Frankfurter Zeitung, 22 de marzo de 1919.

Incluido en GPS, pp. 502-504.

Edición castellana: «A propósito de la investigación sobre la cuestión de la culpa» y «La investigación sobre la cuestión de la culpa», en EP, tomo II, pp. $298-299$ y $300-302$.

1919: «Wissenschaft als Beruf», en Geistige Arbeit als Beruf. Vier Vorträge vor dem Freistudentischen Bund. Erster Vortrag, 1919.

Incluido en WL, pp. 582-613.

Ediciones castellanas:

a) «La ciencia como vocación», en Max Weber, El politico y el científico, Ed. Alianza, Madrid, 1980 (sexta edición, primera en 1967), pp. 180-123; traducción de Francisco Rubio Llotente del original alemán.

b) «La ciencia como vocación», en ESC, pp. 160-192.

c) «La ciencia como vocacion", en Max Weber, El trabajo intelectual como 
«Papers»: Revista de Sociologia

profesión, Ed. Bruguera, Barcelona, 1983; pp. 9.59; traducción del original alemán de Adam Kovacsics Meszaros.

d) Hay también una traducción en Max Weber, El sabio y la politica, Ed. Jorge Alvarez, Buenos Aires, 1972.

1919: «Politik als Beruf», en Geistige Arbeit als Beruf. Vier Vorträge vor dem Freistudentischen Bund. Zweiter Vortrag, 1919.

Incluido en GPS, pp. 505.560.

Ediciones castellanas:

a) «La política como vocación», en Max Weber, El político y el cientifico, Ed. Alianza, Madrid, 1980 (sexta edición, primera en 1967), pp. 81-179; traducción del alemán de Francisco Rubio Lilorente.

b) «La política como vocación», en ESC, pp. 79.159.

c) «La política como vocación», en EP, tomo II, pp. 308-364; se trata de la misma traducción que $\alpha$ ).

d) «La polftica como profesión», en Max Weber, El trabajo intelectual como profesión, Ed. Bruguera, Barcelona, 1983, pp. 61-156; traducción del alemán de Adam Kovacsics Meszaros.

e) Hay también una traducción en: Max Weber, El sabio y la politica, Ed. Jorge Alvarez, Buenos Aires, 1972.

1921: «Die rationalen und soziologiscben Grundlagen der Musik», en Drei

Maskenverlag, Munich, 1921; con una introducción de Theodor Kroyer.

Edición castellana: «Los fundamentos racionales y sociológicos de la música", incluido como apéndice a ES, pp. 1118-1183.

1922: Wirtschaft und Gesellschatt, sección III de Grundriss der Sozialökonomik; publicado póstumamente por Marianne Weber, Tübingen, 1922. Véase en WG los datos de la edición alemana.

Edición castellana:

a) Economia y Sociedad, Ed. Fondo de Cultura Económica, México, 1979 (sexta edición en un solo volumen, primera de 1944); traducción de la cuarta edición alemana (corregida y aumentada) de 1956 por: J. Medina Echavarría, J. Roura Parelle, E. Imaz, E. García Máynez y J. Ferrater Mora. Incluye como apéndice el ensayo de 1921 anteriormente citado.

b) Hay una reproducción de las páginas 5 a 18 de la anterior traducción publicadas bajo el título «Max Weber. Fundamentos metodológicos de la sociologías en Jean-Marie Vincent, La metodologia de Max Weber, Ed. Anagrama, Barcelona, 1972.

c) Se pueden encontrar traducciones parciales de algunos capítulos en ESC según el siguiente orden (hay que señalar que es normal en esta edición que los párrafos aparezcan invertidos y que se interpolen párrafos de orras partes de la obra en el medio de un texto, y todo ello sin que la mayoría de las veces se le advierta al lector):

«Estructuras de poder», en ESC, pp. 159-220 se corresponde a WG, pp. 520530 y a ES, pp. 668-682.

"Clase, status y partido", en ESC, pp. 221-241 se corresponde a WG, páginas $531-540$ y a ES, pp. 682-694. 
«Burocracia» en ESC, pp. 241-199 se corresponde con WG, pp. 551-579 y a ES, pp. 716.752 .

"La sociología de la autoridad carismática" en ESC, pp, 300-309 se corresponde con WG, pp. 654-661 y a ES, pp. 847-856.

«Significado de la disciplina» en ESC, pp. 310-324 se corresponde a WG, pp. $681-687$ y a ES, pp. $882-889$.

d) Traducción parcial de las páginas 1 a 14, realizada por Salvador Giner, publicada en Max Weber. La acción social: ensayos metodológicos, Ediciones Península, Barcelona, 1984, pp. 11-48.

1922: «Die drei reinen Typen der Legitimen Herrschaft», Aus dem Nacblass veröffentlicht, Preussiscbe Jabrbücber, vol. 187, 1922, pp. 1-12.

Edición castellana: ES, pp. 706-716.

1923: Wirtschaftsgeschichte. Abriss der universalen Sozial.und Wirtschaftsgescbichte, S. Hellmann y M. Palyi, Munich, 1923.

Edición castellana: Historis económica general, Ed. Fondo de Cultura Económica, Mađ̃id, 1974 (quinta edición, primera de 1942); traducción del original alemán de Manuel Sánchez Sarto.

Para una mayor facilidad a la hora de cotejar las citas de las obras de Weber reunidas en GPS, GAR y WL con sus correspondientes traducciones, así como la paginación de WG con la de ES, puede consultarse el siguiente cuadto de correspondencias.

\begin{tabular}{|c|c|c|}
\hline GPS & & Ediciones castellanas \\
\hline pp. 1- 25 & ......... & EP, tomo I, pp. 3.29 \\
\hline pp. $26 \cdot 32$ & .......... & Sin traducir \\
\hline pp. 33- 68 & $\ldots \ldots+\cdots$ & $\mathrm{EP}$, tomo XI, pp. 365.402 \\
\hline pp. $\quad 69.111$ & ......... & EP, tomo II, PP. $403-444$ \\
\hline pp. $112-141$ & $\ldots \ldots \ldots$ & Sin traducir \\
\hline pp. 142-145 & ......... & $\mathrm{EP}$, tomo I, pp. $30-34$ \\
\hline pp. $146 \cdot 156$ & .......... & Sin traducir \\
\hline pp. $157-177$ & .......... & $\mathrm{EP}$, tomo I, pp. 35.59 \\
\hline pp. $178-196$ & …...... & Sin ttaducir \\
\hline pp. $197-215$ & …..... & EP, tomo II, Pp. $445-470$ \\
\hline pp. $216-228$ & ......... & Sin traducir \\
\hline pp. 229.232 & ........... & EP, tomo $x$, pp. $163-166$ \\
\hline pp. 233.244 & $\ldots \ldots \ldots$ & Sin traducir \\
\hline pp. 245-291 & ......... & EP, tomo I, pp. $167-217$ \\
\hline pp. $291 \cdot 305$ & ......... & Sin traducir \\
\hline
\end{tabular}


«Papers»: Revista de Sociologia

\begin{tabular}{|c|c|}
\hline pp. $306-443 \ldots \ldots \ldots$ & $\mathrm{EP}$, tomo I, pp. 59.162 \\
\hline pp. $444.447 \ldots \ldots \ldots$ & Sin traducir \\
\hline pp. $448-483$........ & $\mathrm{EP}$, tomo II, pp. $253-293$ \\
\hline pp. $484-487, \ldots \ldots \ldots$ & EP, tomo II, pp. $294-297$ \\
\hline pp. $488.497 \ldots \ldots \ldots$ & Sin traducción \\
\hline p. $498.501 \ldots \ldots \ldots$ & EP, tomo II, pp. $303-307$ \\
\hline pp. $502-504 \ldots \ldots \ldots$ & EP, tomo II, pp. 298-302 \\
\hline pp. $505.560 \ldots \ldots \ldots$ & $\begin{array}{l}\text { EP, tomo II, pp. } 308-364 \text { (consultar al respecto en Ia } \\
\text { bibliografía anterior: } 1919 \text { : «Politik als Beruf»). }\end{array}$ \\
\hline $\begin{array}{l}561.621 \ldots \ldots \ldots \\
\text { (Apéndices) }\end{array}$ & Sin traducir \\
\hline
\end{tabular}

EAR* Ediciones costellanas

\section{Tomo I}

pp. 1-206 ........ ESR, 1, pp. 11-167 (ver referencia completa en la bi. bliografía anterior en 1904-1905)

pp. 207-236 ESR, 1, pp. $169-192$ y ESC, pp. $370-394$

pp. $237-275$ ESR, 1, pp. 193.222

Pp. 276-313 ........ ESR, 1, pp. 223-254

pp. 314.349........ ESR, 1, pp. 255.284

pp. $349-373$........ ESR, 1, Pp. 285-304

pp. 373-395 ........ ESR, 1, pp. 305-322

Pp. $395-430$........ ESR, 1, pp. 323-352

pp. $430-458 \ldots \ldots \ldots$ ESR, 1, pp. 353.375

pp. $458-512$ ….... ESR, 1, pp. $376-418$

pp. $512-536$........ ESR, 1, pp. 419-436

pp. 536.573 ........ ESR, 1, pp. $437-466$ (consultar también la referencia completa en 1916: «Die Wirtschaftsethink...»)

* En el motnento de redactar este repertorio la editorial Taurus anuncia la próxima publicación del tomo I de la Sociología de las religiones (GAR, I), que contendrf́a: "La ética protestante y el espíritu del capitalismo», «Las sectas protestantes y el espíritu del capitalismo» y «La ética económica đe las grazdes religiones I: Confucianismo y Taoismos. Como se ve se corresponde con el contenido del tomo primeto de GAR. Lamentablemente, al no poder disponer aún de dicha traducción, no fue posible introducir aquí los datos correspondientes a traductor y paginación; pese a todo se puede esperar que Taurus, con el rigor que le caracteriza, respete la estructura del original alemán $\mathrm{y}$ nos proporcione una edición digna de un autor como Weber. 
Repertotio bibliográfico castellano de Max Weber

GAR

Ediciones castellanas

Tomo II

pp. 1.31

Sin traducit

pp. $31-48$

ESC, pp. 483-502

pp. $49-109$

Sin traducir

pp. $109-113$

ESC, pp. 502-506

pp. 113.378

Sin traducir

Tomo $I I I$

Totalmente sin traducir

WL

Ediciones castellanas

pp. 1-145

Sin traducir

pp. 146.214

EMS, pp. 39-101

pp. $215-290$

EMS, pp. 102-175

pp. 291.426

Sin traducir

pp. 427-474

EMS, pp. 175-221

pp. 475.488

Sin traducir

Pp. 489.540

EMS, pp. 222-269

pp. 541.581

Se cortesponde con las páginas 5.31 de ES

pp. $582-613$

"La ciencia como vocación». (Véase referencias complepletas en bibliografía 1919: "Wissenschaft als Beruf».)

WG

ES

\section{Halbband}

Erster Teil

Kapitel I

Kapitel II

Kapitel III

Kapitel III.1

Kapitel IIX.2

Kapitel III.3

Kapitel III.4

Kapitel III.S

Kapitel III.6

Kapitel III.7

Kapitel III.8

\begin{tabular}{|c|c|c|c|c|}
\hline 1. 30 & 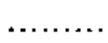 & I & pp. & \\
\hline $31-121$ & & II & pp. & $46-$ \\
\hline $122-176$ & ....... & III & $\mathrm{pp}$. & 170 \\
\hline $122 \cdot 124$ & & III.i, & pp. & $170-$ \\
\hline $125-130$ & & III.ii, & pp. & $173-$ \\
\hline $130-140$ & ... & III.iii, & pp. & 180 \\
\hline 140 & & IIX.iv, & pp. & $193-$ \\
\hline $142-148$ & & III.v, & pp. & $197-$ \\
\hline $148-1$ & & III.vi, & $\mathrm{p}$ & 204 \\
\hline & & & & 214- \\
\hline $158-1$ & & IIX.virii, & & 217 \\
\hline
\end{tabular}


«Papers»: Revista de Sociologia

Kapitel III.9 pp. 167.169

Kapitel III.10 pp. 169-171

Kapitel III.11

Kapite! IV

Zweiter Teil

Kapitel I

Kapitel II

Kapitel III

Kapitel IV

Kapitel V

Kapitel VI pp. $171-176$

pp. $177 \cdot 180$
III.ix, pp. 228- 232

III.x, pp. 232- 235

III.xi, pp. 235- 241

IV pp. 242- 248

Segunda Parte

I pp. $251-272$

II pp. 273- 288

III pp. 289.314

IV Pp. $315-327$

V pp. 328.492

VI pp. $493-497$

\section{Halbbond}

Kapitel VII pp. $387-513 \ldots \ldots \ldots$ VII pp. $498-660$

Kapitel VII pp. $514-540 \ldots \ldots \ldots$ VIII pp. 661.694

Kapitel IX pp. $541-868 \ldots \ldots$ IX $\ldots$ iX $695-1117$

Kapitel IX.1 pp. $541-550 \ldots \ldots \ldots$ IX.i, pp. 695.705

IX.ii, pp. 706- 716

(Este capítulo fue añadido a ES; véase Bibliografía general 1922: «Dei drei...»)

Kapitel IX.2 pp. 551.579 ........ IX.iii, pp. 716- 753

Kapitel IX.3 pp. 580-624 ........ IX.iv, pp. 753-810

Kapitel IX.4 pp. 625-653 ....... IX.v, pp. 810-847

Kapitel IX.5 pp. $654.687 \ldots \ldots \ldots$ IX.vi, pp. $847-889$

Kapitel IX.6 pp. 688-726 ........ IX.vii, pp. $889-938$

Kapitel IX.7 pp. $727.814 \ldots \ldots \ldots$ IX.viii, pp. $938-1047$

Kapitel IX.8 $\quad$ pp. $815-868 \ldots \ldots \ldots$ IX.ix, pp. 1047-1117

3. Holbbond

Sin traducir, es sustituido por un índice temático mucho más escueto. 


\section{Cien tírulos en castellano sobre Weber}

\section{Biografias}

Gerth, H. H. y Wright Mills, C.: «El hombre y su obra. 1. Biografia» en: We. ber, Max; Ensayos de sociología contemporánea, Ed. Martínez Roca, Barcelona, 1972, pp. 11-43.

Honigsheim, Paul: «Recuerdos de Max Weber»; en: Honigsheim, Max Weber, Ed. Paidos, Buenos Aires, 1977, pp. 13-104.

Mitzman, Arthur: La jaula de bierro. Una interpretación bistórica de Max Weber, Ed. Alianza, Madrid, 1976.

Arico, José: «Nota biogxáfica», en Max Weber, Escritos politicos, Ed. Folio, México, 1982, pp. XI-XXIX.

\section{Exposiciones globales}

Aton, Raimond: La sociologia alemana contemporánea, Ed. Paidos, Buenos Aires, $1965^{2}$, pp. 109-169.

Aton, Raimond: Las etapas del pensamiento sociológico, tomo II, Ed. Siglo Veinte, Buenos Aires, 1970, pp. 219-316.

Bendix, Reinhard: Max Weber, Ed. Amorrortu, Buenos Aires, 1979.

Freund, Julien: Sociologia de Max Weber, Ediciones Península, Barcelona, 1967.

Freyer, Hans: La sociología ciencia de la realidad, Ed. Losada, Buenos Aires, 1944, pp. $171-184$ y $228-242$.

Gerth, H. H. y Wright Milis, C.: «Introducción: el hombre y su obra», en Max Weber, Ensayos de sociología contemporánea, Ed. Martínez Roca, Barcelo. na, 1972, pp. 9-94.

Giddens, A.: «El capitalismo y la moderna teoría social», Ed. Labor, Batcelona, 1977, sp. 205-386.

Honigsheim, Paul: «Max Weber sociólogo», en Honigsheim, Max Weber, Ed. Paidos, Buenos Aires, 1977, pp. 117-124.

Honigsheim, Paul: «Max Weber», en Max Weber, Ed. Paidos, Buenos Aires, 1977, pp. 105.115.

Hughes, H. S.: Conciencia y Sociedad, Ed. Aguilar, Madrid, 1972, pp. 205-247.

Marsal, Francisco: Conocer Max Weber y su obra, Ed. DOPESA, Barcelona, 1978.

Parsons, Talcott: La estructura de la acción social, tomo II, Ed. Gredos, Ma. drid, 1968 , pp. 621-864.

Pérez Díaz, V. M.: Introducción a la sociología, Ed. Alianza, Madrid, 1980, pp. 51-80.

Piza, Alfonso: «Enseñanza de Max Weber», en «Revista Colombiana de Sociologían, vol. 1, núm. 1, diciembre 1979, Bogotá, pp. 7-33.

Smelser y Warner: Teoría Sociológica, Ed. Espasa Calpe, Madrid, 1982. 
«Papets»: Revista de Sociologia

Timashelf, N. S.: La teoría sociologica, Ed. Fondo de Cultura Económica, México, 1961, pp. 213.237.

Zeitlin, Irving: Ideología y teoría sociológica, Ed. Amorrortu, Buenos Aires, $1977^{3}$, pp. 127-180.

\section{Aspectos sociológicos generales}

Bendix, Reinhard: «Max Weber y la sociología contemporánea», en Parsons y otros; Presencia de Max Weber, Ed. Nueva Visión, Buenos Aires, 1971, pá. ginas $39-59$.

Dawe, Alan: «La importancia de los valotes» en Sahay, Arun, Max Weber y la sociología moderna, Ed. Paidos, Buenos Aires, 1974, pp. 53-88.

Dahrendotf, Ralf: Oportunidades vitales, Ed. Espasa Calpe, Madrid, 1983, páginas $90-104$ y ss.

Eldrige, J.E. T.: «El eafoque de Weber sobre el estudio socioiógico de los trabajadores industriales», en Sahay, Arun (comp.) Max Weber y la sociologia moderna, Ed. Paidos, Buenos Aires, 1974, pp. 125-142.

Freund, Juliea: Las teorias de las ciencias bumanas, Ediciones Península, Barcelona, 1975, pp. 122-128.

Friedrich, Robert: Sociología de la sociologia, Ed. Amorrortu, Buenos Aires, 1977.

Giner, Saivador: El progreso de la conciencia sociológica, Ed. Ariel, Barcetona, 1974.

González Páramo: «Sociología industrial: aportación de Max Weber», en Revista Internacional de Sociología, núm. 29, enero-marzo, 1979, tomo XXXVII, Madrid, pp. 25.101.

Gurvitch, Georges: Teoria de las clases sociales, Ed. Cuadernos para el diálogo, Madrid, 1971, pp. 139 y ss.

Habermas, I.: Problemas de legitimación en el capitalismo tardío, Ed. Amorrortu, Buenos Aires, 1975, pp. 119 y ss.

Laurin-Frenete, Nicole: Las teorias funcionalistas de las clases sociales. Sociologia e ideología burguesa, Ed. Siglo XXI, Madrid, 1976, pp. 79-117.

Lerena, Carlos: Reprimir y liberar. Crítica sociológica de la educación y de la cultura contemporáneas, Ed. Akal, Madrid, 1983, pp. 449-497.

Lukács, Georg: El asalto a la razón, Ed. Grijalbo, Barcelona, 1976², pp. 485500 .

Lukes, Steven: El individualismo, Ediciones Península, Barcelona, 1975.

Marsal, J. Francisco: Teoria y critica sociológicas, Ed. Guadarrama, Madrid, 1977 , pp. 39 y ss.

Merleau-Ponty, M.: Las aventuras de la dialéctica, Ed. La Pléyade, Buenos Aites, 1974, pp. 13-36.

Moore, Robert: «Historia, economía y religión: una tesis de revisión sobre "las hipótesis de Max Weber" ${ }^{\prime}$, en Sahay, Arun (comp.), Max Weber y la sociologia moderna, Paidos, Buenos Aires, 1974, pp. 107-124. 
Moya, Carios: «Max Weber y la vocación actual de la sociología», en Moya, Sociólogos y sociologia, Ed. Siglo XXI, México, 1975², pp. 113-143.

Nisbet, Robert A.: La formación del pensamiento sociológico I y II, Ed. Amortortu, Buenos Aires, 1969.

Rex, J.: Problemas fundamentales de la teoria sociológica, Ed. Amorrortu, Butenos Aires, 1971.

Rođ̛íguez lbáñez, f. S.: El sueño de la razón, Ed. Taurus, Madrid, 1982.

Schütz, A.: Fenomenología del mundo social, Ed. Paidos, Buenos Aires, 1972. Stark, Wernes: «Max Weber y la heterogonía de los fines», en Parsons y otros, Presencia de Max Weber, Ed. Nueva Visión, Buenos Aires, 1971, pp. 191. 208.

Vericat, J.: Ciencia, Historia y Sociedad, Ed. Istmo, Madrid, 1970.

Veron, Eliseo: Conducta, estructura y comunicación, Ed. Jorge Álvarez, Buenos Aires, 1968, pp. 21-182.

Winch, Peter: Ciencia social y filosofia, Ed, Amorrortu, Buenos Aires, 1972.

\section{Aspectos metodológicos}

Amendola, Giandomenico: Método sociológico e ideología, Ed. A. Redondo, Batcelona, 1973.

Baar, Carl: «Max Weber y el proceso de comprensión de lo social» en Parsons y otros, Presencia de Max Weber, Ed. Nueva Visión, 1971, pp. 207-222.

Beltrán, Miguel: «Acerca de Weber y su ciencia exenta de valoraciones», en Jinénez Blanco y Moya Valgañón, Teorís sociológica contemporánea, Ed. Tecnos, Madrid, 1978, pp. 397-421.

Cerroni, Umberto: Metodología y ciencia social, Ed. Martínez Roca, Barcelona, 1977, pp. 49-54.

Dahrendorf, Ralf: "Ciencia social y juicios de valor», en Dahrendorf, Sociedad y libertad, Ed. Taurus, Madrid, 19712, pp. 36-53.

Gouldner, Alvin W.: «El antiminotatro: el mito de una sociología no valorativan, en Gouldner, La sociologia actual: renovación y critica, Ed. Alianza, Madrid, 1973, pp. 15-35.

Janoska-Bendl, Judith: Max Weber y la sociología de la bistoria. Aspectos metodológicos del tipo ideal, Ed. Sut, Buenos Aires, 1972.

König, René: «El problema de los jujcios de valor en Max Weber» en Talcott Parsons y otros, Presencia de Max Weber, Ed. Nueva Visión, Buenos Aites, 1971 , pp. 73.84 .

Lamo de Espinosa, E.: Juicios de valor y ciencia social, Ed. Fernando Torres, Valencia, 1975.

Mommsen, Wolfgang: «Historia sociológica y sociología histórica» en Mommsen, Max Weber: Sociedad, politica e bistoria, Ed. Alfa, Barcelona, 1981, pp. 213. 244.

Mommsen, Wolfgang: "Comprensión" y "tipo ideal". Acerca de la metodo- 
logía de una ciencia históricas, en Mommsen, Max Weber: Sociedad, politice e bistoria, Ed. Alfa, Barcelona, 1981, pp. 245-274.

Parsons, Talcott: «Evaluación y objetividad en el ámbito de las ciencias socia les: una interpretacion de los trabajos de Max Weber», en Parsons y otros, Presencia de Max Weber, Ed. Nueva Visión, Buenos Aires, 1971, pp. 9-38.

Rex, John: «Tipología y objetividad: un comentario sobre los cuatro métodos sociológicos de Weber», en Sahay (comp.) Max Weber y la sociología moderna, Ed. Paidos, Buenos Aires, 1974, pp. 29.52.

Rossi, Pietro: «Objetividad científica y presupuestos axiológicos» en Parsons y otros: Presencia de Max Weber, Ed. Nueva Visión, Buenos Aires, 1971, pp. 61-71.

Rossi, Pietro: «Introducción» a Max Weber, Ensayos sobre metodología sociológica, Ed. Amorrortu, Buenos Aires, 1978, pp. 9-37.

Runciman, W. C.: Critica de la filosofía de las ciencias sociales de Max Weber, Ed. Fondo de Cultura Económica, México, 1976.

Watkins, J. W. N.: «Tipos ideales y explicación histótica», en A. Rian (ed.), La filosofía de la explicación social, Ed. Fondo de Cultura Económica, Ma. drid, 1976, pp. 130-165.

Sahay, Arun: «La importancia de la metodología de Weber en la explicación sociológica», en Sahay (comp.), Max Weber y la sociología moderna, Ed. Pai. dos, Buenos Aires, 1974, pp. 89-106.

Vincent, Jean-Marie: La metodologia de Max Weber, Ed. Anagrama, Barcelona, 1972.

\section{Racionalidad y racionalización}

Jiménez Blanco, J.: «Weber, Schütz y Garfinkel. Sobre racionalidad» en Jiménez Blanco y Moya Valgañón, Teoría sociológica contemporánea, Ed. Tecnos, Madrid, 1978 , pp. 366-393.

Lamo de Espinosa, E.: La teoría de la cosificación de Marx a la escuela de Frankfort, Ed. Alianza, Madrid, 1981, pp. 85-111.

Marcuse, Herbert: «Industrialización y capitalismo en la obra de Max Weber» en Parsons y otros, Presencia de Max Weber, Ed. Nueva Visión, Buenos Aires, 1971 , pp. 123-145.

Mouzells, Nicos P.; Organización y Burocracia, Ediciones Península, Barcelona, $1975^{2}$.

Pintos de Cea, Juan L.: «Conflicto de racionalidades» en «Ágora», núm. 1, 1981, Universidad de Santiago de Compostela, pp. 65-83.

Saavedra, Luis: «La racionalización en Max Weber» en «Sistema», núm. 42, mayo 1981, Madrid, pp. 93-117.

Bauman, Zygmunt: Pare una sociología crítica, Ed. Marymar, Buenos Aires, 1977. 


\section{Sociología de la religión}

Eisenstadt, S. N.: «La tesis de la Ética Ptotestante» en Robertson (comp.), Sociología de la religión, Ed. Fondo de Cultura Económica, México, 1980, pp. 269-287.

Gabe1, Joseph: «Una lectura marxista de la sociología teligiosa de Max Weber», en Parsons y otros, Presencia de Max Weber, Ed. Nueva Visión, Buenos Aires, 1971, pp. 171-190.

Hill, M.: Sociología de la religion, Es. Cristiandad, Madrid, 1976.

Lenski, Gerhard: El factor religioso, Ed. Labor, Batcelona, 1967.

Matthes, Joachim: Introducción a la sociología de la religión I y II, Ed. Alianza, Madrid, 1971.

\section{Weber, Marx y marxismos}

Feo, Nicola de: Weber y Lukács, Ed. A. Redondo, Barcelona, 1972.

Kozyr-Kowalski, Atanislaw: «Weber y Marx》 en Parsons y otros, Presencis de Max Weber, Ed. Nueva Visión, Buenos Aires, 1971, pp. 243-265.

Lukács, Georg: «Marx y Weber: Reflexiones sobre la decadencia de la ideología”, en Horowitz (comp.), Historia y elementos de la sociología del conocimiento, Ed. EUDEBA, Buenos Aires, 1974', pp. 49-55.

Mommsem, Wolfgang: «Capitalisıno y socialismo. La polémica con Karl Marx», en Mommsen, Max Weber: Sociedad, politica e bistoria, Ed. Alfa, Barcelona, 1981, pp. 169-211.

Runciman, W. G.: «Carlos Marx y Max Weber», en Runcirnan, Ensayos: So. ciología y política, Ed. Fondo de Cultura Económica, México, 1966, pp. 61-87.

\section{Aspectos politicos}

Aron, Raimond: «Max Weber y la política de poder», en «Papers», núm. 15, 1981 (Ed. Barral), Batcelona, pp. 33-54.

Aron, Raimond: «Introducción» a Weber, El político y el científico, Ed. Alian. za, Madrid, 1980', pp. 9-77.

Beetham, David: Max Weber y la teoría politica moderna, Ed. Centro de Es* tudios Constitucionales, Madrid, 1979.

Fallers, L. A.: «El concepto de “autotidad tradicional” en Max Weber», en Parsons y otros, Presencia de Max Weber, Ed. Nueva Visión, Buenos Aires, 1971, pp. 147-169.

Giddens, Anthony: Politica y sociología en Max Weber, Ed. Alianza, Madrid, 1976.

Ginet, Salvador: Sociedad masa: critica del pensamiento conservador, Ed. Península, Barcelona, 1979.

Krygler, Martin: «Weber, Lenin y la realidad del socialismo», en AA.VV., La 
«Papers»: Revista de Sociologia

burocracia, trayectoria de un concepto, Ed. Fondo de Cultura Económica, México, 1981, pp. 117.163.

Mayer, J. P.: Max Weber y la política alemana, Ed. Centro de Estudios Políticos, Madrid, 1966.

Mommsen, W.: «La sociología politica de Max Weber y su filosofía de la historia universal», en Parsons y otros, Presencia de Max Weber, Ed. Nueva Visión, Buenos Aires, 1971, pp. 85-121.

Mommsen, W.: «Pensamiento histórico-universal y pensamiento político», en Mommsen, Max Weber: Sociedad, politica e bistoria, Ed. Alfa, Buenos Aires, Barcelona, 1981, pp. 113-168.

Mommsen, W.: «Max Webex y la crisis del sistema de valores liberal», en Mommsen, «Papers», núm. 15, 1981 (Ed. Barral), Barcelona, pp. 9-32.

Mommsen, W.: «Acerca del concepto de democracia plebiscitaria del líder», en Mommsen, Max Weber: Sociedad, politica e bistoria, Ed. Alfa, Barcelona, 1981, pp. 49-82.

Mommsen, W.: «Los Estados Unidos de Amética», en Mommsen, Max Weber: Sociedad, politica e bistoria, Ed. Alfa, Barcelona, 1981, pp. 83-112.

Mommsen, W.: «Un liberal en la situación límite», en Mommsen, Max Weber: Sociedad, politica e bistoria, Ed. Alfa, Barcelona, 1981, pp. 21-48

Murillo, Francisco: Estudios de sociologia politica, Ed. Tecnos, Madrid, 1972, pp. $146-170$.

Rodríguez-Aguilera Prat, C.: «Metodología y política en Max Weber», en «Sistema», núm. 46, enero de 1982, Madrid, pp. 91-101.

Schapiro, Meyer: «Sobre la política de Max Weber», en Parsons y otros, Presentio de Max Weber, Ed. Nueva Visión, Buenos Aires, 1971, pp. 223-241.

Santiago de Compostela, Diciembre de 1984 